and there was considerable emphysema. On the left side there was a corresponding wound, also, between the seventh and eighth ribs, but higher up and more posterior, situate almost in a direct line from the inferior angle of the scapula; very little air had escaped into the cellular membrane, but on passing the hand over the latissimus dorsi, the air was felt crackling under a considerable portion of that muscle. No doubt could exist as to the lungs being wounded on both sides, and I considered the prognosis as unfavourable as it could well be. As there was no fracture of the ribs, and the wounds were clean, I closed them both with adhesive plaster, applied warmth to the extremities, as he was shivering with cold, and administered some tea.

By nine o'clock, a.m., reaction had taken place; at ten o'clock his pulse had become tolerably steady at 120. Venesection to twenty ounces, which produced faintness; ordered a mixture of spermaceti and ipecacuanha wine to be taken once in three or four hours; to take nothing of any kind except tea and toast and water.

At three, p.m., he was labouring under more severe symptoms; his breathing was much oppressed; he had a good deal of pain and uneasiness about his chest, with a full pulse. Venesection to thirty-four ounces, which made him faint, and on recovering he expressed himself as much relieved.

At nine in the evening his symptoms were again aggravated, but not to so great an extent; his pulse had acquired so much firmness and strength that another blood-letting was readily foreseen, if not then absolutely necessary, and I took thirty ounces before he was faint; I ordered him ten grains of opium and soap pill, and left him for the night, with strict orders to be called if the symptoms returned.

I saw him on Friday morning at six o'clock; he had passed a tolerable night, and his state presented no symptoms which demanded interference. As I was desirous of obtaining some information about the wounds of the lungs, I removed one strip of plaster, and found that the air was passing with much less freedom than twenty-four hours ago, although the external wounds, in consequence of the continued oozing of blood, were not healing by the first intention. The wounds were again immediately closed; one ounce of castor oil was administered. Mr. R. Smith, the senior surgeon of the infirmary, saw him to-day; he considered he would have a hard struggle for it, and recommended a small abstraction of blood in the evening.

Eight, p.m. Has continued up to this time pretty much as in the morning; bowels relieved. I bled him to ten ounces; repeated the opium pill.

Saturday morning. He has passed a tolerable night again, and is free from pain; pulse soft, but quick. He takes the spermaceti mixture, from which he says he finds much relief and comfort. No air whatever passes through the wounds.

Sunday morning. He remains in a quiet state; the giant has evidently been conquered since Thursday evening. The external wounds are not at all disposed to heal, but those of the lungs are apparently closed. Allowed a small teacupful of thin bread and milk, which is the first food he has taken.

From this time he slowly but gradually progressed, and he left his bed about three weeks after the injury. The bloody expectoration continued for five or six days, and the emphysema gradually subsided. The wound on the right side of the chest did not heal for a month. Adhesion of the pleura on the right side has taken place to a considerable extent, covering a space as large as the hand; on the left side the state of the parts is not so easily ascertained, owing to the thick layer of muscle.

He subsequently was blistered, and took iodine for some time. He remains in the force on reserve duty, which does not expose him to cold or wet. He has had several attacks of shortness of breathing and pain in the side, but they have all given way to a day's confinement in bed, and a mixture of tartar emetic These attacks have latterly been less frequent. $\mathrm{He}$ has got married, and at the time of my writing this he tells me his health has much improved within the last twelvemonth.

\section{CASE OF PURPURA,} WITH

\section{DESTRUCTIVE DISEASE OF THE ANCLE-} JOINT.

By F. A. Bulley, Esq.,

Surgeon to the Royal Berkshire Hospital, Reading.

On March 14, 1842, I attended the inspection of the body of a poor boy, 17 years old, who had died the day before, from a disease of the ancle-joint, for which he had been treated seventeen weeks, and, as far as I could learn, his complaints had existed for some time prior to that time. His case not having been under my immediate care, I am unable to detail its progress ; but the patient seemed to be of a highly scrofulous constitution, and of a very delicate frame of body, and had latterly become hectic, and greatly emaciated by his disease. Some time ago, before his health had become too much affected by the local disease, amputation had been proposed to him; but to this he obstinately refused to assent, and it was observed that he became worse after the proposal had been made, from the effect the fear of it had upon his enfeebled nervous system, which, naturally weak, had become weaker through the wearing nature of his complaints. Latterly his legs had become nearly covered with minute purpurous spots, as well as the surface of his body, where there were some petechial stains, but not nearly so numerous or distinct as on the extremities.

Inspection.-The lungs were of a healthy appearance externally, and there was no appearance of any tubercular disease of their structure. They were somewhat darker coloured than natural, from congestion of their vessels. There was rather more fluid than common in the pericardium. The heart was preternaturally small, not exceeding in bulk that of a child of six years' old; but it did not present the shrivelled and wrinkled appearance of its exterior, described by $\mathrm{Dr}$. Hope, to indicate that it had ever been of greater size. The auricles were somewhat dilated, and, together with the ventricles and large vessels emanating from the heart, were filled with dark-coloured fluid blood, which, when removed, had an unhealthy, treacly appearance, and seemed to have entirely lost its power of coagulating. The muscular structure of the body was pale and wasted. There were no appearances of petechial spots on any part of the serous tissues of the thoracic or abdominal cavities or viscera.

Examination of the Diseased Ancle.-On the inner side of the leg, over and just above the ancle-joint, there were two or three ragged openings of a dark black colour, from which, during life, had flowed a dark-coloured ichorish fluid, but no pus. From these openings a probe could not be directly introduced into the cavity of the joint, owing to their not being immediately placed over the apertures in the capsular ligament, but by passing it underneath the skin for a short distance through the disorganised cellular tissue, the opening was readily discovered; the distance of the subcutaneous passage was, of course, greatest in those openings farthest from the joint. The integument over the inner projecting surface of the internal unciform bone was rather more dark-coloured than the rest, and an aperture in the skin over it was somewhat larger than the others, and through this a 
roughness from necrosis of the surface of this bone was perceptible.

The connecting and capsular ligaments generally of the ancle-joints were thickened, and of a dark, unhealthy colour, from the rupture of some vessels in their structure, from which blood had escaped, and there had seemingly been a passive hæmorrhage from these vessels for some time prior to his death. The synovial lining membrane had entirely disappeared. There was no appearance of any pus in the joint. The cartilage of the articulating portions of the astragalus and tibia had become absorbed, leaving a surface of smooth bone of a very dark and unhealthy colour, being deeply injected with dark-coloured blood, but not necrosed; on these surfaces there was a thin layer of brownish, tenacious, coagulated lymph, of an offensive smell, otherwise the interior of the joint had a dry appearance. The end of the tibia, for about an inch and a half, was in a state of high vascular congestion, and extremely soft, so as to be easily cut with a scalpel, as well as the astragalus and other bones of the tarsus, but their articulations with each other, with the exception of the ancle-joint, were not obviously affected, their cartilages and ligaments being entire, and apparently in no way affected by the disease.

\section{REMARKS.}

The foregoing case is not entirely wanting in pathological interest. It is, I believe, not unusual in purpura for hæmorrhage from the gums and mucous membranes to coexist with petechial spots on the various cuticular surfaces of the body; but in this instance there had been no epistaxis, nor had any hæmorrhagic tendency manifested itself in any other than the integumentary structure. The entire absence of the simultaneous appearance of the petechial disease in the serous parenchymatous tissue is also, I believe, uncommon. Perhaps, however, the exceeding smallness of the heart is deserving the greatest consideration. I have observed that in several cases of cachectic disease which I have had an opportunity of inspecting after death, the heart has been found to be preternaturally small; and I have lately been disposed to attribute many of the symptoms observed during life to this imperfect development of the organ.

The feebleness of the circulation, the pale and cachectic appearance, the excitability or depression of the general nervous energy, the disposition to diseases of the joints and the denser structures of the body, from the want of sufficient power in the heart, from its small size, to propel its blood into the intimate capillary system of these tissues, by which their healthy innervation is disturbed, and they are rendered more liable to morbid change-lead me to think that many of the signs and appearances of scrofulous and other diseases of diminished vitality, are frequently dependent upon this abnormal condition. From what this arrest of development primarily results, it is diffi. cult exactly to say, since the other organs of the body are often in such cases fully formed, and capable, considering the error in the circulating centre, of performing their functions comparatively well. It has struck me that it may take place in this way. A child may be born of unhealthy or half-starved parentsborn, as it were, with its blood vitiated on its very entrance into life; subsequent imperfect nourishment from the mother in infancy, and afterwards in early youth, may keep up this impoverished condition of the blood for many years. I assume that in such a case the circulating fluid, thus altered in its chemical and vital properties, is incapable of healthily stimulating the nervous and muscular systems, and that thus the heart, not being roused to proper continuous action by the stimulus of the blood, like other muscles not duly exercised, ceases to enlarge, and thus its development becomes prematurely and permanently arrested. I may mention, that $I$ have never before this had an opportunity of so fully investigating the pathology of purpura, so that I cannot say of my own experience whether the heart is usually found to be small in these cases; but $I$ have read of instances where it has been so, and particularly of one related by Dr. Johnston, in the "Cyclopædia of Practical Medicine," where, after death, the organ was found of an extremely small size; which leads me to think that such imperfect development of this organ is not rare in this disease.

In the dissection of the joint there is nothing particularly worthy of observation. The vascular and softened state of the bones, in the neighbourhood of joints affected with scrofulous disease, has been so often and so minutely described, that the appearances in this case will be at once recognised as belonging to that particular class described by Sir B. Brodie and other authors, with this exception, that instead of the cancellous structure of the bones being filled, as is usual, with cheesy matter, it was filled with semifluid blood, the result, probably, of exhalation from vessels under the same state of disease as had affected the cuticular textures of the body.

\section{CASE OF VENOUS HAMORRHAGE FROM}

A N A B SCESS.

By Robert Storrs, Esq., Doncaster.

As the following case bears some analogy to the one of Mr. Liston, lately discussed at the MedicoChirurgical Society, it may not prove unexceptable to the readers of the "Provincial Journal."

William Heaton, a strong muscular man, aged 36 , by trade a master shoemaker, was seized, Dec. 14, 1839, with severe febrile symptoms, accompanied by an extensive diffused swelling over the front of the throat, affecting its cellular texture from the chin to the chest, of a phlegmonous erysipelatous character. In spite of leeches, fomentations, poultices, \&c., and other local and general treatment, suppuration commenced, and on the 21st, evident fluctuation could be felt on the left side of the larynx. As the constitutional symptoms were very severe, I opened the abscess immediately, and above a pint of excessively offensive and sanious pus was discharged, accompanied with some large shreds of what appeared then to be dead cellular structure. From this opening, which was a very free one, an immense discharge took place, at a moderate computation, about a pint or more daily; and the sac of the abscess was found to extend itself from the left of the larynx and trachea, to the nape of the neck on that side, and from the angle of the jaw to the clavicle. The discharge of pus continued without abatement, and of a most offensive smell; severe symptoms of constitutional irritation and of exhaustion came on, and he required the constant administration of wine and of opiates.

On the 30th some hæmorrhage occurred from the opening, evidently of venous blood, which was con. 